\title{
Extrapolation of Survival Benefits in Patients with Transthyretin Amyloid Cardiomyopathy Receiving Tafamidis: Analysis of the Tafamidis in Transthyretin Cardiomyopathy Clinical Trial
}

Benjamin Li (D) · Jose Alvir · Michelle Stewart

Received: April 13, 2020 / Published online: June 10, 2020

(C) The Author(s) 2020
Digital Features To view digital features for this article go to https://doi.org/10.6084/m9.figshare.12310772 .

Electronic supplementary material The online version of this article (https://doi.org/10.1007/s40119. 020-00179-2) contains supplementary material, which is available to authorized users.

B. Li $(\bowtie) \cdot J$. Alvir

Pfizer, New York, NY, USA

e-mail: Benjamin.Li@pfizer.com

M. Stewart

Pfizer, Groton, CT, USA generalized F) were applied to systematically fit different candidate curves to existing patientlevel data from the 30-month treatment period in ATTR-ACT. The relative goodness-of-fit for each candidate curve was then tested by Akaike's and Bayesian information criteria to select a single model that was fitted to the placebo and pooled tafamidis treatment arms.

Results: A gamma distribution was selected as best fitting model and fitted to both treatment arms. The resulting estimated median overall survival was 35.16 months for placebo and 52.64 months for tafamidis (difference 17.48 months).

Conclusions: This extrapolation of survival data from ATTR-ACT further supports the efficacy of tafamidis in patients with ATTR-CM. Owing to the limitations of this analysis, these survival estimates should be interpreted with caution; however, they are consistent with recently presented findings from a combined analysis of data from ATTR-ACT and interim data from an ongoing long-term extension study (median follow-up 36 months; ClinicalTrials.gov number NCT02791230).

Trial Registration: ClinicalTrials.gov: NCT019 94889.

Keywords: Amyloid; Cardiomyopathies; Heart failure; Survival; Tafamidis 


\section{Key Summary Points}

Why carry out this study?

Patients with transthyretin amyloid cardiomyopathy (ATTR-CM) in the placebo and tafamidis arms of ATTR-ACT did not achieve median overall survival by the completion of the study at 30 months. A statistical extrapolation method was developed to estimate their survival beyond the 30 -month trial duration.

\section{What was learned from the study?}

Findings from the survival extrapolation analysis suggest that at least $50 \%$ of patients receiving tafamidis would survive for approximately 53 months, compared to 35 months for those receiving placebo, providing further support for the efficacy of tafamidis in ATTR-CM.

\section{INTRODUCTION}

Transthyretin amyloid cardiomyopathy (ATTR$\mathrm{CM}$ ) is a progressive, life-threatening disease caused by the aggregation of amyloid fibrils in the heart. These amyloid fibrils are formed by misfolded transthyretin (TTR) protein [1]. Deposition of misfolded TTR monomers or oligomers in the myocardium results in restrictive cardiomyopathy and heart failure, and accumulation in the conduction system may lead to bundle-branch or atrioventricular block and atrial arrhythmias [2]. ATTR-CM is primarily a condition affecting older adults and can have a genetic (hereditary; ATTRv) or nongenetic (wild-type; ATTRwt) basis [3]. Diagnosis is often substantially delayed after symptom onset, and estimated median survival is 2.5 years and 3.6 years after diagnosis of ATTRv and ATTRwt, respectively $[4,5]$.

Tafamidis is a benzoxazole derivative that lacks nonsteroidal anti-inflammatory drug activity and binds with high affinity and selectivity to TTR, inhibiting amyloid fibril formation [6]. Tafamidis is currently approved in more than 40 countries worldwide to delay the progression of peripheral neurologic impairment in transthyretin amyloid polyneuropathy, and was recently approved in the USA, Canada, Japan, and Brazil for the treatment of ATTR-CM of both types (ATTRv and ATTRwt). In the Tafamidis in Transthyretin Cardiomyopathy Clinical Trial (ATTR-ACT), this TTR stabilizer was shown to be statistically superior to placebo over 30 months of treatment in reducing allcause mortality and cardiovascular-related hospitalizations, with a reported 30\% lower risk of both outcomes [7].

In the completed 30-month study, median overall survival (mOS) was not achieved in either treatment arm of ATTR-ACT, with 57.1\% of patients in the placebo arm and $70.5 \%$ of patients in the tafamidis arm surviving at the end of the trial, limiting full assessment of the potential survival benefit of treatment [7]. We therefore developed a statistical extrapolation method to estimate survival with tafamidis versus placebo in patients with ATTR-CM beyond the 30-month duration of ATTR-ACT.

\section{METHODS}

The ATTR-ACT was a phase 3, placebocontrolled, double-blind, randomized trial (ClinicalTrials.gov number NCT01994889) in which 177 patients received placebo and 264 patients with ATTR-CM received treatment with tafamidis ( 80 or $20 \mathrm{mg}$ ) for 30 months [7]. In the primary analysis of ATTR-ACT findings, the investigators hierarchically assessed all-cause mortality, followed by the frequency of cardiovascular-related hospitalizations, over the 30-month study. Findings were compared between the placebo group and the pooled tafamidis (80 and $20 \mathrm{mg}$ ) treatment group. Heart transplantation and implantation of a cardiac mechanical assist device (i.e., left-ventricle assist device), as indicators of end-stage disease, were treated as equivalent to death in this analysis (as in the primary ATTR-ACT analysis). 
The method used for our analysis adhered to the technical support guidelines issued by the National Institute for Health and Care Excellence on survival analysis extrapolation with patient-level data [8]. To initiate the model selection process, log-cumulative hazard plots (and variations) were constructed to visually verify whether assumptions for multiple models held true. The models included exponential, Weibull, gamma, log-logistic, log-normal, Gompertz, generalized gamma, and generalized $F$ models. (Assumptions for these models are summarized in Electronic Supplementary Material [ESM] Table S1.)

Plots of the curves for candidate models (i.e., those for which assumptions were not violated) were compared via visual inspection with Kaplan-Meier curves based on existing individual patient-level data from the four treatment arms (i.e., placebo, tafamidis $80 \mathrm{mg}$, tafamidis $20 \mathrm{mg}$, and pooled tafamidis $80 \mathrm{mg}$ and $20 \mathrm{mg}$ ) in the 30-month treatment period in ATTR-ACT to determine how well each model fit the data. The relative goodness-of-fit of each candidate curve was subsequently tested using Akaike's information criterion (AIC) and the Bayesian information criterion (BIC) [9]. Based on these fit criteria, the best-fitting single model was selected and fitted to data from the placebo group and the pooled tafamidis 20-mg and 80-mg dose group in ATTR-ACT. The other models (i.e., those not selected based on fit criteria) were similarly fitted to these data for comparison purposes.

This article is based on previously conducted studies and does not contain any studies with human participants or animals performed by any of the authors. All patient data are anonymized with no personal data, so informed consent was not required.

\section{RESULTS}

The top best-fitting candidate models were selected for each of the four treatment arms based on the AIC/BIC goodness-of-fit testing (Table 1). The gamma distribution was the only top model shared across all four treatment arms; the data did not suggest that other models were substantially different within each treatment arm. The gamma distribution model was selected as the best-fitting model and was fitted to the placebo and pooled tafamidis treatment arms in ATTR-ACT.

According to output from the gamma model, the extrapolated time when survival probability was approximately 0 ranged from 22 years in

Table 1 Testing goodness-of-fit of each candidate curve using Akaike's information criterion and Bayesian information criterion for each treatment arm

\begin{tabular}{|c|c|c|c|c|c|c|c|c|}
\hline \multirow[t]{2}{*}{ Model } & \multicolumn{2}{|l|}{ Placebo } & \multicolumn{2}{|c|}{ Pooled tafamidis } & \multicolumn{2}{|c|}{ Tafamidis $80 \mathrm{mg}$} & \multicolumn{2}{|c|}{ Tafamidis $20 \mathrm{mg}$} \\
\hline & $\overline{\mathrm{AIC}}$ & BIC & $\overline{A I C}$ & BIC & $\overline{\text { AIC }}$ & BIC & $\overline{\text { AIC }}$ & BIC \\
\hline Exponential & 770.26 & 773.44 & 854.33 & 857.91 & 587.82 & 590.99 & 268.27 & 270.74 \\
\hline Weibull & 754.50 & 760.86 & 852.85 & 860.00 & 586.89 & 593.23 & 269.61 & 274.57 \\
\hline Gamma $^{a}$ & $\underline{755.92}$ & $\underline{762.27}$ & $\underline{852.45}$ & $\underline{859.60}$ & $\underline{586.70}$ & $\underline{593.04}$ & $\underline{269.44}$ & $\underline{274.40}$ \\
\hline Log-logistic & 756.27 & 762.62 & 851.90 & 859.05 & 586.48 & 592.82 & 269.11 & 274.06 \\
\hline Log-normal & 765.21 & 771.57 & 850.99 & 858.15 & 587.05 & 593.39 & 267.74 & 272.69 \\
\hline Gompertz & 754.16 & 760.51 & 855.10 & 862.26 & 588.31 & 594.65 & 270.24 & 275.19 \\
\hline Generalized gamma & 755.80 & 765.33 & 852.94 & 863.67 & 588.43 & 597.94 & 268.61 & 276.04 \\
\hline Generalized F & 757.77 & 770.47 & 854.94 & 869.24 & 590.30 & 602.99 & 270.61 & 280.52 \\
\hline
\end{tabular}

$A I C$ Akaike's information criterion, BIC Bayesian information criterion

Values in bold denote top candidate models for each treatment arm

${ }^{a}$ Underling indicates the top model (Gamma) shared by all four treatment arms based on AIC or BIC criteria 


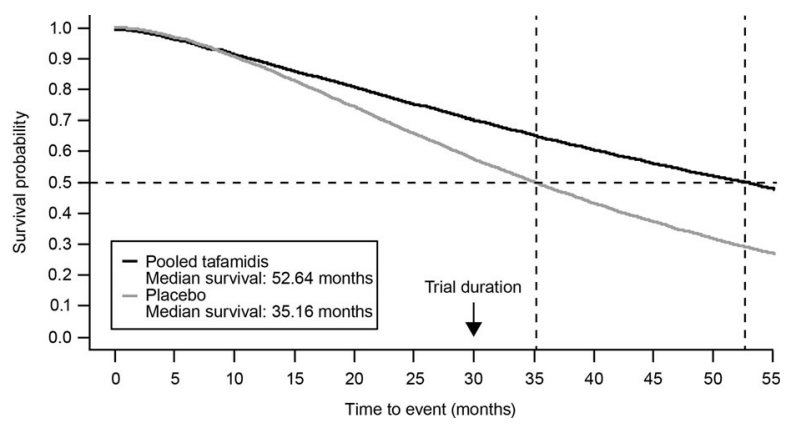

Fig. 1 Gamma model fitted to patient-level data for allcause mortality

the placebo group to 44 years in the pooled tafamidis group. The resulting estimated mOS was 35.16 months for placebo and 52.64 months for the pooled tafamidis group, a difference of 17.48 months (Fig. 1). (Corresponding findings from the other models are shown, along with those from the gamma model, in ESM Fig. S1.)

\section{DISCUSSION}

Based on the published Kaplan-Meier curves for mortality during the 30-month ATTR-ACT study, the mOS was not reached in either the placebo or pooled tafamidis treatment groups [8]. Therefore, we used a statistical method based on extrapolation of observed study data to estimate patients' mOS after the study end at 30 months. Our extrapolation method applied a best-fit model approach extending beyond 30 months and evaluated the goodness-of-fit of the models to the observed ATTR-ACT data. The extrapolated curves were based on fitting parametric curves to the data; two standard criteria were used to evaluate the model fit and complexity-AIC and BIC-with lower values determining best fit. After assessing the estimated curves and AIC/BIC, we selected the gamma model as the best fitting model and used it to estimate mOS beyond the 30-month trial duration. We estimated that at least $50 \%$ of patients receiving tafamidis would live approximately 53 months, versus 35 months for patients receiving placebo, a 17.5-month longer estimated mOS with tafamidis compared with placebo.

Interestingly, our extrapolated results appear to be consistent with the recently presented findings of a combined analysis of data from ATTR-ACT [7] and interim data from an ongoing, open-label, long-term extension study of ATTR-ACT (ClinicalTrials.gov number NCT02791230; data cutoff: February 2018) [10]. In the latter extension study, patients who received tafamidis meglumine during ATTRACT continued to receive the agent at the same dose, whereas patients who received placebo were randomized to receive either tafamidis meglumine $20 \mathrm{mg}$ or $80 \mathrm{mg}$. (Data from the two tafamidis dose groups were pooled, as in the primary analysis [7].) Across the two studies, after a median follow-up of 36 months, the risk of all-cause mortality was significantly lower in the tafamidis/tafamidis group than in the placebo/tafamidis group [10], and the survival curves for each group over time approximated those obtained in the current extrapolation analysis.

The extrapolation approach used for our analysis has limitations that should be considered when interpreting findings. Because the study duration of ATTR-ACT was 30 months, the accuracy of the extrapolated results cannot be fully evaluated based solely on data from ATTR-ACT. In addition, the extrapolation model did not control for the covariates in ATTR-ACT (e.g., New York Heart Association class). Finally, alternative model approaches (e.g., finite mixture models) could result in different estimates of the mOS. As a result of these limitations, the survival estimates derived from this extrapolation approach are best interpreted as an approximation of the potential benefit of tafamidis.

\section{CONCLUSIONS}

Future results of the ongoing, long-term extension study to ATTR-ACT will provide additional information about the survival benefit of tafamidis in patients with ATTR-CM. However, because patients receiving placebo during the prior 30-month treatment period were switched 
to treatment with tafamidis upon entering the study extension, a direct comparison of tafamidis and placebo will be difficult, especially in the long term. In this case, extrapolation may again be a valuable tool to estimate differences in mOS between patients receiving tafamidis and those who received placebo (i.e., in the absence of long-term placebo data).

\section{ACKNOWLEDGEMENTS}

Funding. This study was sponsored by Pfizer, which also funded the journal's Rapid Service fee.

Authorship. All named authors meet the International Committee of Medical Journal Editors (ICMJE) criteria for authorship for this article, take responsibility for the integrity of the work as a whole, and have given their approval for this version to be published.

Medical Writing and Editorial Assistance. Medical writing support for this article was provided by Donna McGuire of Engage Scientific Solutions, and was funded by Pfizer.

Prior Presentation. This manuscript is based on work previously presented at the 2019 Heart Failure Society of America (HFSA) 23rd Annual Scientific Meeting, which was held on 13-16 September 2019, in Philadelphia, Pennsylvania, USA.

Disclosures. Benjamin Li, Jose Alvir, and Michelle Stewart are full-time employees of Pfizer and hold Pfizer stock and/or stock options.

Compliance with Ethics Guidelines. This article is based on previously conducted studies and does not contain any studies with human participants or animals performed by any of the authors. All patient data are anonymized with no personal data, so informed consent was not required.
Data Availability. Upon request, and subject to certain criteria, conditions, and exceptions (see https://www.pfizer.com/science/clinicaltrials/trial-data-and-results for more information), Pfizer will provide access to individual de-identified participant data from Pfizer-sponsored global interventional clinical studies conducted for medicines, vaccines, and medical devices (1) for indications that have been approved in the USA and/or EU or (2) in programs that have been terminated (i.e., development for all indications has been discontinued). Pfizer will also consider requests for the protocol, data dictionary, and statistical analysis plan. Data may be requested from Pfizer trials 24 months after study completion. The de-identified participant data will be made available to researchers whose proposals meet the research criteria and other conditions, and for which an exception does not apply, via a secure portal. To gain access, data requestors must enter into a data access agreement with Pfizer.

Open Access. This article is licensed under a Creative Commons Attribution-NonCommercial 4.0 International License, which permits any non-commercial use, sharing, adaptation, distribution and reproduction in any medium or format, as long as you give appropriate credit to the original author(s) and the source, provide a link to the Creative Commons licence, and indicate if changes were made. The images or other third party material in this article are included in the article's Creative Commons licence, unless indicated otherwise in a credit line to the material. If material is not included in the article's Creative Commons licence and your intended use is not permitted by statutory regulation or exceeds the permitted use, you will need to obtain permission directly from the copyright holder. To view a copy of this licence, visit http://creativecommons.org/licenses/by$\mathrm{nc} / 4.0 /$.

Open Access. This article is licensed under a Creative Commons Attribution-NonCommercial 4.0 International License, which permits any non-commercial use, sharing, adaptation, distribution and reproduction in any medium or format, as long as you give appropriate credit to the original author(s) and the source, provide 
a link to the Creative Commons licence, and indicate if changes were made. The images or other third party material in this article are included in the article's Creative Commons licence, unless indicated otherwise in a credit line to the material. If material is not included in the article's Creative Commons licence and your intended use is not permitted by statutory regulation or exceeds the permitted use, you will need to obtain permission directly from the copyright holder. To view a copy of this licence, visit http://creativecommons.org/licenses/bync/4.0/.

\section{REFERENCES}

1. Rapezzi C, Quarta CC, Riva L, et al. Transthyretinrelated amyloidoses and the heart: a clinical overview. Nat Rev Cardiol. 2010;7:398-408.

2. Ruberg FL, Berk JL. Transthyretin (TTR) cardiac amyloidosis. Circulation. 2012;126:1286-300.

3. Maurer MS, Hanna M, Grogan M, et al. Genotype and phenotype of transthyretin cardiac amyloidosis: THAOS (Transthyretin Amyloid Outcome Survey). J Am Coll Cardiol. 2016;68:161-72.

4. Lane T, Fontana M, Martinez-Naharro A, et al. Natural history, quality of life, and outcome in cardiac transthyretin amyloidosis. Circulation. 2019;140:16-26.

5. Grogan M, Scott CG, Kyle RA, et al. Natural history of wild-type transthyretin cardiac amyloidosis and risk stratification using a novel staging system. J Am Coll Cardiol. 2016;68:1014-20.

6. Bulawa CE, Connelly S, Devit M, et al. Tafamidis, a potent and selective transthyretin kinetic stabilizer that inhibits the amyloid cascade. Proc Natl Acad Sci USA. 2012;109:9629-34.

7. Maurer MS, Schwartz JH, Gundapaneni B, et al. Tafamidis treatment for patients with transthyretin amyloid cardiomyopathy. N Engl J Med. 2018;379: 1007-166.

8. Latimer N. NICE DSU Technical Support Document 14: Survival analysis for economic evaluations alongside clinical trials - extrapolation with patientlevel data (2011). https://nicedsu.org.uk/wpcontent/uploads/2016/03/NICE-DSU-TSD-Survivalanalysis.updated-March-2013.v2.pdf. Accessed 12 June 2019.

9. Collett D. Modelling survival data in medical research. 2nd ed. Boca Raton: Chapman \& Hall/ CRC; 2003.

10. Elliott P, Drachman BM, Gottlieb SS, et al. Interim analysis of data from a long-term, extension trial of tafamidis meglumine in patients with transthyretin amyloid cardiomyopathy. Eur Heart J. 2019;40(Suppl 1):ehz480011 (abstract 1169). 